\title{
Papers
}

\section{Psychiatric disorders and risky sexual behaviour in young adulthood: cross sectional study in birth cohort}

\author{
Sandhya Ramrakha, Avshalom Caspi, Nigel Dickson, Terrie E Moffitt, Charlotte Paul
}

\begin{abstract}
Objective To determine if risky sexual intercourse, sexually transmitted diseases, and sexual intercourse at an early age are associated with psychiatric disorder.

Design Cross sectional study of a birth cohort at age 21 years with assessments presented by computer (for sexual behaviour) and by trained interviewers (for psychiatric disorder).

Setting New Zealand in 1993-4.

Participants 992 study members (487 women) from the Dunedin multidisciplinary health and development study. Complete data were available on both measures for 930 study members.

Main outcome measures Psychiatric disorders (anxiety, depression, eating disorder, substance dependence, antisocial disorder, mania, schizophrenia spectrum) and measures of sexual behaviour. Results Young people diagnosed with substance dependence, schizophrenia spectrum, and antisocial disorders were more likely to engage in risky sexual intercourse, contract sexually transmitted diseases, and have sexual intercourse at an early age (before 16 years). Unexpectedly, so were young people with depressive disorders. Young people with mania were more likely to report risky sexual intercourse and have sexually transmitted diseases. The likelihood of risky behaviour was increased by psychiatric comorbidity.

Conclusions There is a clear association between risky sexual behaviour and common psychiatric disorders. Although the temporal relation is uncertain, the results indicate the need to coordinate sexual medicine with mental health services in the treatment of young people.
\end{abstract}

\section{Introduction}

The onset of psychiatric disorders and risky sexual behaviour both peak in young adulthood. ${ }^{12}$ Although it is generally accepted that both problems have important public health implications, ${ }^{3}$ little is known about the relation between the two. We examined whether psychiatric disorders and risky sexual behaviour occur in the same individuals and, if so, whether there are specific relations. Information about concurrence of mental health disorders and risky sexual behaviour can be used to identify populations at risk for both problems and may help to inform prevention and intervention practices.

Previous studies of clinic and general population samples have reported associations between psychiatric problems and risky sexual behaviour and sexually transmitted diseases. ${ }^{5-9}$ Most of these studies, however, used highly selected samples or relied on global indicators of psychiatric status. Additionally, most studies have concentrated solely on substance use. Other common major psychiatric disorders, such as anxiety and depression, have rarely been considered. Those studies that have used representative samples and standardised measures of psychiatric disorders have focused on a restricted range of sexual health outcomes (for example, HIV). As a result of these limitations the existing findings are less than optimal for informing clinical practice or public health policy. Indeed, most studies of psychiatric disorders tend not to examine sexual behaviour in the same sample and vice versa.

We examined the links between a range of psychiatric problems and aspects of sexual behaviour at age 21 years using data from an epidemiological study of an unselected birth cohort.

\section{Method}

Participants

Participants were members of the Dunedin multidisciplinary health and development study, ${ }^{10}$ which investigates the health and behaviour of a birth cohort born during a one year period between 1 April 1972 and 31 March 1973 in Dunedin, a city of about 120000 on New Zealand's South Island. The cohort was established when the children were three years old and were traced for follow up; $91 \%$ of the eligible children (still resident in the province) participated in the assessment. We used data gathered at the assessment at age 21 years when $97.3 \%(n=992,487$ women $)$ of the living study members participated. Data on mental health were collected for 961 study members and data on sexual behaviour for 935 study members, with complete data available for 930. Ethical approval was received, and confidentiality was guaranteed for each component of the assessment.

\section{Measurement of psychiatric disorder}

Data on mental health were collected in a private interview by using the diagnostic interview schedule. ${ }^{11}$ Its

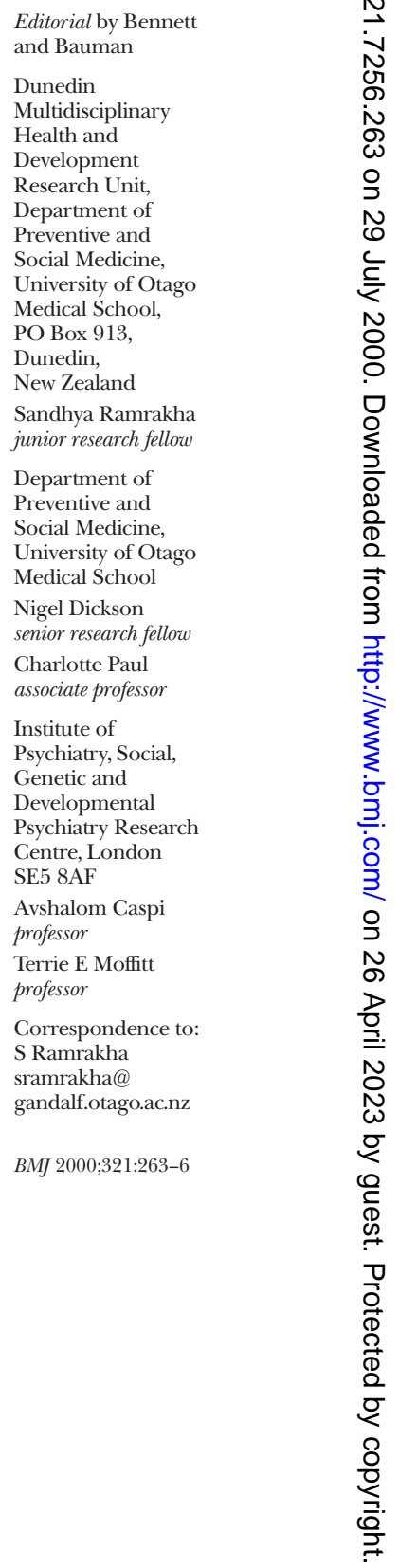


procedures, reliability, validity, and descriptive epidemiology in this sample have been described elsewhere. ${ }^{12}$ Using a reporting period of the past year, we assessed the following disorders according to Diagnostic and Statistical Manual of Mental Disorders, third edition, revised (DSM-III-R) ${ }^{13}$ : anxiety (which included any of social phobia, simple phobia, agoraphobia, generalised anxiety, and panic disorder), depression (major depressive disorder or dysthymia, or both), eating disorder (anorexia or bulimia, or both), substance dependence (marijuana or alcohol, or both), antisocial disorder (antisocial personality or residual conduct disorder, or both), mania, and schizophrenia spectrum. This spectrum category included individuals who responded "yes, definitely" to questions about the positive symptoms of schizophrenia, including bizarre beliefs such as being "sent special messages through the television or radio" and sensory perceptions such as "hearing voices other people could not hear." Any symptoms with plausible explanations or that occurred solely under the influence of drugs or during a major depressive episode were ruled out. Structured psychiatric interviews are known to identify people who endorse psychotic type experiences and beliefs, some of whom have clinical psychosis but many of whom do not, ${ }^{14}$ and whose symptoms lie on a continuous spectrum from schizotypal personality disorder to schizophrenia. We expect only one quarter of our spectrum cases to develop schizophrenia, but their mental health was clearly impaired: $85 \%$ had comorbid diagnoses, $77 \%$ reported that the symptoms interfered with their lives, $54 \%$ had corroboration from an informant, 39\% had contact with treatment, and 10\% were prescribed psychiatric medication, though none was admitted to hospital that year. ${ }^{12}$

\section{Measurement of sexual behaviour}

Sexual behaviour in the previous 12 months was assessed with a questionnaire based on the 1990 British national survey of sexual attitudes and lifestyles. ${ }^{15}$ Questions were presented by computer, with an interviewer present who could not see the subjects' responses but was available to assist. We used the following measures to assess sexual behaviour and outcomes.

Risky sexual intercourse-Those who reported having sexual intercourse with three or more partners in the past year and who "never or only sometimes" used a condom were defined as engaging in risky sexual behaviour. Condom use reduces the risk of sexually transmitted diseases, ${ }^{16}$ and there is a definite increase in the incidence of sexually transmitted diseases in those who have had three or more partners. ${ }^{17}$

Sexually transmitted diseases-Study members were introduced to questions about sexually transmitted diseases with the statement: "There are some diseases, or infections, that can be passed on during sex. These are called sexually transmitted diseases or STDs." Common examples were given, and those who responded positively were questioned on how often this had occurred in their life (recurrent episodes of the same infection were treated as one disease).

Early sexual intercourse-Study members were asked about their age at first intercourse. Study members who had sexual intercourse before the age of 16 years were considered to have had early sexual intercourse. Their characteristics have been reported elsewhere. ${ }^{18}$

\section{Statistical analysis}

To examine the relation between psychiatric disorder and risky sexual behaviour and outcomes we used $2 \times 2$ contingency tables (disorder versus no disorder and risky sexual behaviour versus no risky sexual behaviour) and obtained risk ratios and 95\% confidence intervals. All risk ratios were adjusted with the Mantel-Haenszel test to take account of sex difference in the prevalence of disorder or risky sexual behaviour variables. Adjustment for socioeconomic status was also investigated. The socioeconomic status of the study members' families was coded into six categories according to occupation. ${ }^{19}$ To examine whether people with more than one psychiatric problem were more likely to have risky sexual intercourse we used multivariate logistic regression. The cumulative risk associated with psychiatric comorbidity was tested by estimating the linear combination of coefficients and standard errors.

Using moderated regression analyses we also tested whether the links between psychiatric disorder and risky sex differed for men and women and for people from different socioeconomic backgrounds. To do this we computed a multiplicative interaction term between each of the disorders and sex (or socioeconomic status) and then estimated hierarchical logistic regression analyses for each of the outcomes of risky behaviour. Disorder and sex (or socioeconomic status) were entered on the first step and the interaction term on the second step. Significant interaction terms indicate that the links between psychiatric disorders and risky sexual behaviour vary across different groups.

\section{Results}

Table 1 shows the links between psychiatric disorders and risky sexual intercourse, sexually transmitted diseases, and early sexual intercourse. In the first row prevalence of outcomes for study members who were free from disorder are presented; they represent the control group against which disorder groups are compared. The tests for sex showed that these links did not differ significantly for men and women, so our data are combined in table 1. (Prevalence rates of psychiatric disorders ${ }^{12}$ and sexual outcomes ${ }^{17} 18{ }^{20}$ have previously been reported by sex for this sample.) In addition, the links were not moderated by socioeconomic statusthat is, the links reported in table 1 were the same, and obtained equally, across all socioeconomic groups.

Compared with people with no psychiatric disorder study members with anxiety disorders were more likely to report sexually transmitted diseases. Study members with depressive disorders, substance dependence, and antisocial disorders were more likely to engage in risky sexual intercourse and report sexually transmitted diseases and were younger at first sexual intercourse. If study members were diagnosed with mania they were more likely to have engaged in risky sexual intercourse and to report sexually transmitted diseases. Finally, those with symptoms in the schizophrenia spectrum were more likely to engage in risky sexual intercourse and report sexually 
Table 1 Relation between psychiatric disorders and sexual behaviour outcomes at age 21 in a birth cohort. Figures are adjusted risk ratios (95\% confidence intervals). All comparisons are between diagnostic group named versus those with no disorder in the sample

\begin{tabular}{|c|c|c|c|c|c|c|}
\hline \multirow{2}{*}{$\begin{array}{l}\text { DSM-III-R diagnostic } \\
\text { comparison group at age } 21 \text { (No } \\
\text { of participants) }\end{array}$} & \multicolumn{2}{|c|}{$\begin{array}{l}\text { Risky sexual intercourse in past year } \\
\qquad(n=153)\end{array}$} & \multicolumn{2}{|c|}{$\begin{array}{c}\text { Lifetime history of sexually transmitted } \\
\text { diseases }(n=104)\end{array}$} & \multicolumn{2}{|c|}{$\begin{array}{c}\text { Sexual intercourse before age } \\
16 \text { years }(n=274)\end{array}$} \\
\hline & No & Risk ratios* $(95 \% \mathrm{Cl})$ & No & Risk ratios* $(95 \% \mathrm{CI})$ & No & Risk ratios* $(95 \% \mathrm{Cl})$ \\
\hline No psychiatric disorder (572) & 70 & & 46 & & 132 & \\
\hline Anxiety disorders (128) & 16 & $1.2(0.7$ to 2.0$)$ & 20 & $1.6(1.0$ to 2.6$) \dagger$ & 37 & $1.1(0.8$ to 1.6$)$ \\
\hline Depressive disorders (172) & 42 & $2.2(1.5$ to 3.1$) \dagger$ & 25 & $1.6(1.0$ to 2.5$) \dagger$ & 53 & $1.3(1.0$ to 1.6$) \dagger$ \\
\hline Eating disorders (13) $\ddagger$ & 3 & 2.7 (0.9 to 7.6$)$ & 1 & $0.7(0.1$ to 4.9$)$ & 7 & $2.2(1.3$ to 3.6$) \dagger$ \\
\hline Substance disorders (155) & 51 & $2.4(1.8$ to 3.3$) \dagger$ & 27 & $2.6(1.7$ to 4.0$) \dagger$ & 72 & $2.1(1.7$ to 2.6$) \dagger$ \\
\hline Antisocial disorders (57) & 19 & $2.4(1.6$ to 3.7$) \dagger$ & 9 & $3.2(1.6$ to 6.5$) \dagger$ & 30 & $2.8(2.0$ to 3.8$) \dagger$ \\
\hline Manic episode (19) & 6 & $2.5(1.2$ to 5.0$) \dagger$ & 7 & $4.4(2.3$ to 8.3$) \dagger$ & 6 & $1.3(0.7$ to 2.6$)$ \\
\hline Schizophrenia spectrum (39) & 10 & $2.1(1.2$ to 3.7$) \dagger$ & 7 & $2.3(1.1$ to 4.8$) \dagger$ & 20 & $2.3(1.6$ to 3.2$) \dagger$ \\
\hline
\end{tabular}

DSM-III-R=Diagnostic and Statistical Manual of Mental Disorders, third edition, revised.

*There were no significant sex differences in associations between disorder and sexual behaviour variables. Risk ratios were adjusted to take account of sex differences in prevalence of disorder or sexual behaviour variables.

$\dagger P<0.05$.

$\ddagger$ Significant link with eating disorders and early sexual intercourse should be interpreted with caution as cell sizes were small.

transmitted diseases and were younger at first sexual intercourse.

Table 2 shows the associations between comorbid psychiatric conditions and risky sexual behaviour. We examined the comorbidity of the three most common psychiatric disorders that are linked with risky sexual behaviour (depression, substance dependence, and antisocial disorders). Comorbid psychiatric conditions show stronger associations with risky behaviour compared with any single psychiatric disorder.

\section{Discussion}

The results provide evidence that psychiatric disorders and risky sexual behaviour occur in the same people at age 21 with unusual prevalence. Although risky sexual behaviour and sexually transmitted diseases are common among this age group, a disproportionate burden is carried by those with psychiatric disorders. The associations were not moderated by sex. This effect does not seem to be due to social disadvantage in the family of origin because adjustment for socioeconomic background made no difference to the results. Even if the associations were partly mediated by other aspects of social or educational background, the primary association is important clinically. Young people with psychiatric disorder function with more difficulty in society than those without disorder. ${ }^{12}$ The damaging consequences to health of risky sexual behaviour, such as unplanned pregnancy and sexually transmitted diseases, compound the impairments those with psychiatric problems already experience. Psychiatric comorbidity, which occurs in half of psychiatric cases ${ }^{12}{ }^{21}$ and is associated with increased risk of impairment, ${ }^{22}$ signals additional sociosexual risk.

Our results confirm previous findings of an association between sexual risk taking and externalising problems such as conduct disorder and substance abuse. $^{5-7}$ The strongest association of risky sexual behaviour was with disorders characterised by disinhibition or a pattern of impulsive behaviour (for example, antisocial personality, mania, and drug and alcohol dependence). Two additional findings are noteworthy. Firstly, young adults who present with symptoms in the schizophrenia spectrum were more likely to take part in risky sexual intercourse, develop sexually transmitted diseases, and have sexual intercourse at an early age. Secondly, depression, the most common psychiatric problem in the population, was also linked with these three outcomes. Furthermore, depression combined with an antisocial disorder or substance disorder increased the risk. This link is noteworthy as rates of depression escalate from age 15 to 21 years ${ }^{23}$ the period when sexual activity likewise emerges. About one third of people who use primary care services exhibit depressive symptoms. ${ }^{24}$ On the one hand, depressed young adults may engage in risky sexual behaviour (and substance abuse) because of feelings of hopelessness, worthlessness, and disregard for self or in an attempt to self treat their depression. On the other hand, harmful consequences from a sexual relationship may precipitate a depressive episode.

We did not assess the full spectrum of DSM-III-R disorders, making it possible that we underestimated the extent of concurrence of psychiatric disorders and risky sexual behaviour. We also examined the concurrence of psychiatric disorders and sexual behaviour cross sectionally. To inform prevention and intervention efforts, longitudinal analyses are needed to determine the temporal nature of this association, which may vary by disorder. Bad sexual experiences may lead to depression, but antisocial personality may lead to spreading sexually transmitted diseases.

To our knowledge this is the first report that establishes strong links between a wide range of psychiatric disorders and sexual behaviour. Prevalence and incidence rates of psychiatric disorders and sexual behaviour in the Dunedin sample are similar to those

Table 2 Relation between psychiatric comorbidity and sexual behaviour outcomes at age 21 in birth cohort. Figures are adjusted risk ratios* (95\% confidence intervals). All comparisons are between psychiatric comorbid groups named versus those with no disorder in sample. Base rates of comorbid cases within mania and schizophrenia spectrum groups were too low for reliable analyses

\begin{tabular}{|c|c|c|c|}
\hline $\begin{array}{l}\text { DSM-III-R diagnostic } \\
\text { comorbid comparison group } \\
\text { at age } 21 \text { (No of participants) }\end{array}$ & $\begin{array}{l}\text { Risky sexual } \\
\text { intercourse in } \\
\text { past year }\end{array}$ & $\begin{array}{l}\text { Lifetime history of } \\
\text { sexually transmitted } \\
\text { diseases }\end{array}$ & $\begin{array}{l}\text { Sexual intercourse } \\
\text { before age } 16 \text { years }\end{array}$ \\
\hline $\begin{array}{l}\text { Depressive disorders }+ \\
\text { substance disorders }(n=50)\end{array}$ & 3.7 (2.6 to 5.4$) \dagger$ & 2.5 (1.4 to 4.6$) \dagger$ & $1.6(1.1$ to 2.4$) \dagger$ \\
\hline $\begin{array}{l}\text { Depressive disorders + } \\
\quad \text { antisocial disorders }(n=17)\end{array}$ & 3.7 (2.1 to 6.4$) \dagger$ & $4.5(1.9$ to 10.7$) \dagger$ & 2.6 (1.6 to 4.2$) \dagger$ \\
\hline $\begin{array}{l}\text { Substance disorders + } \\
\text { antisocial disorders }(n=32)\end{array}$ & 3.6 (2.3 to 5.6$) \dagger$ & 4.1 (1.9 to 8.5$) \dagger$ & 2.8 (2.0 to 3.9$) \dagger$ \\
\hline
\end{tabular}

DSM-III-R=Diagnostic and Statistical Manual of Mental Disorders, third edition, revised.

${ }^{*}$ Risk ratios were adjusted to take account of sex differences in prevalence of disorder or sex variables. $\dagger \mathrm{P}<0.05$. 


\section{What is already known on this topic}

Onset of psychiatric disorders and risky sexual behaviour both peak in young adulthood

\section{What this study adds}

A disproportionate burden of risk and disease associated with sexual behaviour is borne by young people with psychiatric problems

Depression, substance dependence, antisocial personality, mania, and schizophrenia spectrum are associated with risky sexual behaviour and sexually transmitted diseases

Psychiatric comorbidity increases the likelihood of sexual risk taking

found among young adults in the United States and United Kingdom, ${ }^{12}{ }^{20}$ thereby lending confidence to the generalisability of the data. The results show that the most common psychiatric disorders in young people (substance dependence and depression) are the disorders that are linked to sociosexual problems. The findings highlight the need to coordinate sexual medicine with mental health services in the treatment of young people. Awareness of this potential comorbidity may also assist with prevention strategies. Moreover, early detection will be facilitated if practitioners are aware that risky sexual behaviour may be associated with mental health problems.

We are grateful to Dr P A Silva, founder of the Dunedin study, and the study members and their families for their long term involvement. We thank Dr Richie Poulton, director of the Dunedin study, for his valuable comments on earlier drafts of this paper.

Contributors: SR participated in the study design, analysed and interpreted the data, and wrote the first draft of the paper. AC coordinated the collection of the mental health data, participated in the study design, and assisted with the analysis and interpretation of the data and writing of the paper. ND coordinated the collection of the sexual health data, participated in the study design, and critically edited the paper. TEM coordinated the collection of the mental health data, participated in the study design, and assisted with the interpretation of the data and writing of the paper. CP participated in the design of the study, provided advice on interpretation of the results, and revised the paper. SR, AC, and ND are guarantors of the study.

Funding: Health Research Council of New Zealand, US National Institute of Mental Health (MH-4507, MH-49414, MH-56344), and UK Medical Research Council. The Dunedin Multidisciplinary Health and Development Research Unit is supported by the Health Research Council of New Zealand.

Competing interests: None declared.

1 Institute of Medicine, Committee on Prevention of Mental Disorders, Division of Biobehavioral Sciences and Mental Disorders. Reducing risk for mental disorders: frontiers for preventive research. Washington, DC: National Academy Press, 1994

2 Centers for Disease Control and Prevention. Trends in sexual risk behaviour among high school students-United States, 1991-1997. MMWR $1998 ; 47: 749-52$

3 World Health Organization. The world health report 1998: life in the 21st century. A vision for all. Report of the Director General. Geneva: World Health Organization, 1998.

4 Murray CJ, Lopez $\mathrm{AD}$, eds. The global burden of disease: a comprehensive assessment of mortality and disability from diseases, injuries and risk factors in 1990 and projected to 2020. Cambridge, MA: Harvard University Press, 1996.

5 Baker DG, Mossman D. Potential HIV exposure in psychiatrically hospitalized adolescent girls. Am J Psychiatry 1991;148:4:528-30.

6 Bardone AM, Moffitt TE, Caspi A, Dickson N, Silva PA. Adult mental health and social outcomes of adolescent girls with depression and conduct disorder. Dev Psychopathol 1996;8:811-29.

7 Cooper ML, Peirce RS, Huselid RF. Substance use and sexual risk taking among black adolescents and white adolescents. Health Psychol 1994;13:251-62.

8 Lowry R, Holzman D, Truman BI, Kann L, Collins JL, Kolbe LJ. Substance use and HIV-related sexual behaviour among US high school students: are they related? Am J Public Health 1994;84:1116-20.
9 Adcock AG, Nagy S, Simpson JA. Selected risk factors in adolescent suicide attempts. Adolescence 1991;104:817-28.

10 Silva PA, Stanton WR, eds. From child to adult: the Dunedin multidisciplinary health and development study. Auckland: Oxford University Press, 1996.

11 Robins LN, Helzer JE, Coughan J, Ratcliff KS. National Institute of Mental Health diagnostic interview schedule: its history, characteristics, and validity. Arch Gen Psychiatry 1981:38:381-9.

12 Newman DL, Moffitt TE, Caspi A, Magdol L, Silva PA, Stanton WR. Psychiatric disorder in a birth cohort of young adults: prevalence, comorbidity, clinical significance, and new case incidence from ages 11 to 21.J Consult Clin Psychol 1996;64:552-62.

13 American Psychiatric Association. Diagnostic and statistical manual of mental disorders ( $3 r d$ ed, rev). Washington, DC: American Psychiatric Association, 1987.

14 Kendler KS, Gallagher TJ, Ableson JM, Kessler RC. Lifetime prevalence, demographic risk factors and diagnostic validity of nonaffective psychosis as assessed in a community sample. Arch Gen Psychiatry 1996;53:1022-31.

15 Johnson AM, Wadsworth J, Wellings K, Field J. Sexual attitudes and lifestyle. Oxford: Blackwell, 1994

16 Centers for Disease Control and Prevention. Update: barrier protection against HIV infection and other sexually transmitted diseases. MMWR 1993;42:589-91.

17 Dickson N, Paul C, Herbison P, McNoe B, Silva PA. The lifetime occurrence of sexually transmitted diseases among a cohort aged 21. NZ Med J 1996;109:308-12

18 Paul C, Fitzjohn J, Herbison P, Dickson N. The determinants of sexual intercourse before age 16 in a birth cohort.J Adolesc Health 2000;27:136-47.

19 Elley WB, Irving JC. A socioeconomic index for New Zealand based on levels of education and income from the 1966 census. $N$ Z J Educational Studies 1972;7:153-67.

20 Dickson N, Paul C, Herbison P, Silva PA. First sexual experience: age, coercion, and later regrets reported by a birth cohort. BMJ 1998;316:29-33.

21 Kessler RC, McGonagle KA, Zhao S, Nelson CB, Hughes M, Eshleman S, et al. Lifetime and 12 month prevalence of DSM-III-R psychiatric disorders in the United States: results from the national comorbidity study. Arch Gen Psychiatry 1994;51:8-19.

22 Newman DB, Moffitt TE, Caspi A, Silva PA. Comorbid mental disorders: implications for treatment and sample selection. J Abnormal Psychol 1998:107:305-11.

23 Hankin BL, Abramson LY, Moffitt TE, Silva PA, McGee R, Angell KE. Development of depression from preadolescence to young adulthood: emerging gender differences in a 10-year longitudinal study. J Abnormal Psychol 1998;107:128-40.

24 Üstün TB, Sartorius N. Mental illness in general health care: an international study. Chichester: World Health Organization, 1995.

(Accepted 25 April 2000)

\section{Corrections and clarifications}

Prospective investigation of transfusion transmitted infection in recipients of over 20000 units of blood In this article by Fiona A M Regan and colleagues (12 February, pp 403-6), the time span of the study was not clear. In the second sentence of the Methods section, the dates given should have included the years (1 August 1991 to 31 May 1996).

$A B C$ of arterial and venous disease: swollen lower limb (part 1-general assessment and deep vein thrombosis) Two errors occurred in this article by $\mathrm{W}$ Peter Gorman and colleagues (27 May, pp 1453-6). In the algorithm (p 1454), a small but important word ("or") was missing: the right hand arrow from the box "Consider clinical probability" should go to "Moderate or high" (not just "High"). Under the section on treatment ( $\mathrm{p}$ 1455) the fifth sentence of the second paragraph should read: "The activated partial thromboplastin time should be checked six hourly until the target is reached and then daily to maintain the ratio [not the international normalised ratio] of activated partial thromboplastin time to control at 1.5 to 2.5."

Measuring performance in the NHS: what really matters?

A small typographical error crept into this article by John Appleby and Andrew Thomas (27 May, pp 1464-7). In the second paragraph in the box entitled "Healthcare resource groups" the second sentence should have started: "For example, category H17 is "soft tissue or other bone procedures ..." (not "soft tissue for bone procedures") 